\title{
Molecular loops in the Galactic centre; evidence for magnetic floatation accelerating molecular gas
}

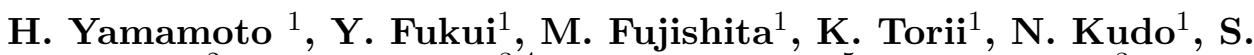

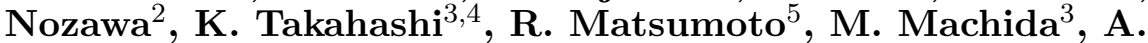 \\ Kawamura $^{1}$, Y. Yonekura ${ }^{6}$, N. Mizuno ${ }^{1}$, T. Onishi ${ }^{1}$ and A. Mizuno \\ ${ }^{1}$ Dep. of Astrophysics, Nagoya Univ., 464-8602, Japan ${ }^{2}$ Dep. of Science, Ibaraki Univ., \\ 310-8512, Japan ${ }^{3}$ NAOJ, 118-8588, Japan ${ }^{4}$ The Graduate Univ. for Advanced Studies, \\ 240-0193, Japan ${ }^{5}$ Dep. of Physics, Faculty of Science, Chiba Univ., 263-8522, Japan ${ }^{6}$ Dep. of \\ Physics Science, Osaka Prefecture Univ., 599-8531, Japan ${ }^{7}$ Solar-Terrestrial Environment Lab., \\ Nagoya Univ., 464-8601, Japan E-mail:hiro@a.phys.nagoya-u.ac.jp
}

The new molecular image obtained by NANTEN telescope in the galactic center has revealed the existence of the two loop like structures, loop 1 and loop 2, which have never been seen before toward $l=355^{\circ}$ to $358^{\circ}$. The velocities of loop 1 and loop 2 are -180 to $-90 \mathrm{~km} \mathrm{~s}^{-1}$ and -90 and $-40 \mathrm{~km} \mathrm{~s}^{-1}$, respectively, and these two loops have strong velocity gradients. The foot points of the loops show a very broad linewidth of $\sim 40$ to $80 \mathrm{~km} \mathrm{~s}^{-1}$ whose large velocity spans are characteristic of the molecular gas near the galactic center. Therefore, we classified the loops as being located in the galactic center and adopt a distance of $8.5 \mathrm{kpc}$. Then, the projected lengths of loop 1 and loop 2 were estimated as $\sim 500$ and $\sim 300 \mathrm{pc}$, respectively and velocity gradients corresponds to $\sim 80$ $\mathrm{km} \mathrm{s}^{-1}$ per $250 \mathrm{pc}$ along loop 1 and $\sim 60 \mathrm{~km} \mathrm{~s}^{-1}$ per $150 \mathrm{pc}$ along loop 2 . The heights of these loops are also estimated as $\sim 220$ to $\sim 300$ pc from the galactic plane, significantly higher than the typical scale height in the nuclear disk.

Each of the loops has a mass of $\sim 0.8 \times 10^{5} \mathrm{M}_{\odot}$ as a lower limit by combining the ${ }^{12} \mathrm{CO}$ and ${ }^{13} \mathrm{CO}$ data and assuming local thermodynamical equilibrium at $50 \mathrm{~K}$. The kinetic energy in a loop was estimated to be $\sim 0.9 \times 10^{51} \mathrm{erg}$ for a velocity dispersion of $30 \mathrm{~km} \mathrm{~s}^{-1}$. The velocity and the energy of loops can not be explained by a super nova. Therefore, we offer a model incorporating MHD instability to explain the formation of the two loops. From this model we found that two model loops calculated by an MHD code were a good match for the observations. We used parameters of $100 \mathrm{~cm}^{-3}$ in gas number density and $150 \mu \mathrm{m}$ in magnetic field. Then, the Alfven speed was calculated as $24 \mathrm{~km} \mathrm{~s}^{-1}$.

This model offers naturally significant heating of the warm molecular gas at the foot points. The velocity dispersion of the broad CO features corresponds to kinetic temperature higher than about $10^{4} \mathrm{~K}$ if the shock is completely converted into thermal energy at the foot points. We suggest that the present model has the potential to be applied to the other salient broad velocity features in the galactic center and to the heating of the molecular gas at their foot points.

\section{Acknowledgements}

The NANTEN project is based on a mutual agreement between Nagoya Univ. and the CIW. We appreciate the hospitality of the members of the LCO. We thank Japanese public donors and companies for the contribution to the project. This work is financially supported in part by a Grant-in-Aid for Scientific Research from the Ministry of Edu., Culture, Sports, Sci. and Tech. of Japan (No. 15071203) and from JSPS (No. 14102003, core-to-core program 17004). 